Notfall Rettungsmed 2016 $\cdot 19: 496-499$ DOI 10.1007/s10049-016-0216-z Online publiziert: 1. September 2016 (c) Springer-Verlag Berlin Heidelberg 2016

CrossMark
K. Singler, ${ }^{1,2}$ H. Dormann ${ }^{3} \cdot$ C. Dodt ${ }^{4} \cdot$ H. J. Heppner ${ }^{5,6} \cdot$ R. Püllen $^{7} \cdot$ M. Burkhardt ${ }^{9} \cdot$ W. Swoboda ${ }^{8} \cdot$ R. E. Roller-Wirnsberger ${ }^{10} \cdot$ G. Pinter ${ }^{11} \cdot$ P. Mrak $^{12} \cdot$ T. Münzer $^{13}$ ${ }^{1}$ Institut für Biomedizin des Alterns, Friedrich-Alexander-Universität Erlangen-Nürnberg, Nürnberg, Deutschland

${ }^{2}$ Klinik für Geriatrie, Klinikum Nürnberg, Paracelsus Medizinische Privatuniversität, Nürnberg, Deutschland ${ }^{3}$ Zentrale Notaufnahme, Klinikum Fürth, Fürth, Deutschland

${ }^{4}$ Notfallzentrum, Städtisches Klinikum München, Bogenhausen, München, Deutschland

${ }^{5}$ Klinik für Geriatrie, Helios Klinik Schwelm, Schwelm, Deutschland

${ }^{6}$ Private Universität Witten/Herdecke gGmbH, Witten, Deutschland

${ }^{7}$ Medizinisch-Geriatrische Klinik, Agaplesion Markus Klinik, Frankfurt am Main, Deutschland

${ }^{8}$ Akutgeriatrie, Klinikum Main-Spessart, Marktheidenfeld, Deutschland

${ }^{9}$ IV. Medizinische Klinik, Geriatrie, Universitätsklinikum Mannheim, Mannheim, Deutschland

${ }^{10}$ Professur für Geriatrie and kompetenzbasierte Curriculumsentwicklung, Abteilung für Innere Medizin, Medizinische Universität Graz, Graz, Österreich

${ }^{11}$ Akutgeriatrie, Remobilisation, Klinikum Klagenfurt am Wörthersee, Klagenfurt am Wörthersee, Österreich

${ }^{12}$ Abteilung für Innere Medizin und Akutgeriatrie/Remobilisation, Landeskrankenhaus Voitsberg, Voitsberg, Österreich

${ }^{13}$ Geriatrische Klinik, Kompetenzzentrum Gesundheit und Alter, St. Gallen, Schweiz

\section{Der geriatrische Patient in der Notaufnahme}

\section{Positionspapier der Deutschen Gesellschaft interdisziplinäre Notfall- und Akutmedizin (DGINA), der Deutschen Gesellschaft für Geriatrie (DGG), der Deutschen Gesellschaft für Gerontologie und Geriatrie (DGGG), der Österreichischen Gesellschaft für Geriatrie und Gerontologie (ÖGG) und der Schweizerischen Fachgesellschaft für Geriatrie (SFGG)}

\section{Hintergrund}

„Die Menschen werden immer älter“ [1]. Die Gründe für die steigende Lebenserwartung sind multifaktoriell: anhaltend niedrige Geburtenraten, gestiegener Wohlstand, Verbesserung der Ernährungssituation und der hygienischen Verhältnisse sowie stetige Entwicklungen im Bereich der Medizin.

2007 wurden von den deutschsprachigen geriatrischen Fachgesellschaften und 2008 auf EU-Ebene Definitionen er- arbeitet, welche den "geriatrischen Patienten“ charakterisieren [2-4]. Diese beziehen sich nicht auf das chronologische Alter, sondern auf die mit dem Alter einhergehende Multimorbidität, die alterstypisch erhöhte Vulnerabilität mit Gefahr der Chronifizierung und dem erhöhten Risiko des Autonomieverlusts [2, 4].

Der demographische Wandel betrifft alle Bereiche der Gesundheitsversorgung und es werden v. a. die Notaufnahmen als Akut- und Notfallversorger vor große Herausforderungen gestellt werden [5].
Epidemiologische Angaben über den Anteilälterer Patienten in Notaufnahmen stammen meist aus dem angloamerikanischen Raum. In einem Übersichtsartikel von Amizadeh et al. wird der Anteil älterer Notaufnahmepatienten ( $\geq 65$ Jahre) mit bis $\mathrm{zu} 20 \%$ angegeben [6]. Im deutschsprachigen Raum gibt es aktuell nur eine Umfrage der Arbeitsgruppe „Der ältere Patient in der Notfallmedizin“ (ÄlPaNo) der DGINA. Diese beziffert den Anteil der über 70-Jährigen in 
deutschen Notaufnahmen im Mittel bei $30 \%$ [7].

\section{Der geriatrische Patient - im Notfall ein Hochrisikopatient}

Eine qualitativ hochwertige, sektorenübergreifende notfallmedizinische Versorgung älterer Patienten muss sichergestellt werden. Trotz der steigenden Zahl dieser Patientengruppe und deren speziellen Bedürfnissen gibt es derzeit keine deutschsprachigen Handlungsempfehlungen für den Bereich der Notaufnahmen, obwohl diese dringend benötigt werden.

Die zur Vorstellung in der Notaufnahme führenden Problemstellungen sind auch aufgrund der Komorbiditäten komplex. Bedingt durch die erhöhte Vulnerabilität sind ältere multimorbide Patienten, z. B. mit vorhandenen geriatrischen Syndromen, im Gegensatz zu Jüngeren häufig stärker von Akuterkrankungen betroffen. Sie zeigen eine höhere Behandlungsdringlichkeit. Die Triage und Erstuntersuchung dieser Patienten birgt bei oftmals unspezifischer und atypischer Symptomatik zusätzlich verschiedene Fallstricke [8-10].

$35 \%$ der Patienten haben vorbestehende akustische, visuelle und kognitive Einschränkungen $[11,12]$. Diese können, wenn sie die eigenständige Informationsweitergabe durch den Patienten zum Behandlungszeitpunkt beeinflussen, zu einem großen Informationsdefizit führen [12-14]. Ein häufig bestehendes Delir, Prävalenz ca. $14 \%$, aggraviert die Situa-

Die Autoren sind am Positionspapier für die folgenden Gesellschaften beteiligt:

Arbeitsgemeinschaft derältere Patient in der Notfallmedizin (ÄIPaNo) der DGINA: PD Dr. K. Singler

Deutsche Gesellschaft für interdisziplinäre Notfallmedizin (DGINA): Prof. Dr. H. Dormann, Prof.Dr.C.Dodt

Deutsche Gesellschaft für Geriatrie (DGG): PD Dr. K. Singler, Prof. Dr. H. J. Heppner, PD Dr. R. Püllen

Deutsche Gesellschaft für Gerontologie und Geriatrie (DGGG): Dr. W. Swoboda, PD Dr. H. Burkhardt

Österreichische Gesellschaft für Geriatrie und Gerontologie (ÖGG): Prof. Dr. R. Roller Wirnsberger

Schweizerische Fachgesellschaft für Geriatrie (SFGG):PD Dr. T.Münzer tion zusätzlich und wird meist nicht erkannt [12, 15-17].

Die aufgrund der Multimorbidität bestehende Polymedikation erhöht oftmals die Vulnerabilität dieser Patienten und ist nicht selten der Grund für die bestehende Symptomatik. Daher müssen sowohl Polymedikation, wie auch funktionelle, kognitive und soziale Aspekte zwingend systematisch erfasst werden [18].

Diese Faktoren tragen dazu bei, dass im Gegensatz zu jüngeren Patienten die Verweildauer geriatrischer Patienten in der Notaufnahme häufig deutlich länger ist $[19,20]$.

Ältere Patienten werden häufiger stationär aufgenommen, da es den Notaufnahmen an geeigneten Möglichkeiten fehlt. Zustand und Lebensqualität verschlechtern sich; die Patienten sind häufiger von externen Hilfen abhängig [6, 21]. Die Vorstellung in einer Notaufnahme mit nicht auf geriatrische Bedürfnisse abgestimmten Strukturen und Prozessen ist für die Patienten als Hochrisikosituation zu sehen und geht in der Folge mit einer Verschlechterung der Selbsthilfefähigkeit und erhöhten Mortalität einher [6].

\section{Der Anspruch - Qualität}

Um eine qualifizierte Versorgung geriatrischer Patienten in der Notaufnahme zu gewährleisten, bedarf es angemessener personeller Ressourcen, fachspezifischen Wissens und Strukturen, welche eine lückenlose sektorenübergreifende medizinische und pflegerische Versorgung sicherstellen können. Kennzahlen und Qualitätsindikatoren hierfür sind in der internationalen Literatur bislang unterrepräsentiert [22-24]. Lediglich für einzelne Teilbereiche, wie z. B. Patienten mit kognitiven Einschränkungen, liegen aktuell Struktur und Prozessindikatoren vor [25, 26]. Auch das Flaggschiff der externen Qualitätssicherung im Gesundheitswesen in Deutschland, der „Qualitätsreport“, welcher mehr als 450 Qualitätsindikatoren aus 31 Leistungsbereichen umfasst, schließt diese Lücke nicht $[27,28]$.

Unterstützt durch die DGINA-Arbeitsgemeinschaft „Der ältere Patient in der Notfallmedizin“ (ÄlPaNo) konnten in den letzten Jahren mittels der durch das Institut für Qualität und Patientensicherheit (BQS) empfohlenen QUALIFYMethode im Rahmen des GeriQ-Projekts Qualitätsindikatoren für die Versorgung älterer Patienten in der Notaufnahme entwickelt werden $[29,30]$.

Somit wird erstmals für Deutschland ein Katalog mit Qualitätsindikatoren für die optimale Versorgung geriatrischer Notfallpatienten zur Verfügung stehen.

Dieser umfasst 32 Struktur-, 30 Prozess- und 5 Ergebnisindikatoren, von denen folgende vorrangig umgesetzt werden müssen:

- obligatorischer Ausschluss eines

Delirs in der Notaufnahme,

- Identifizierung eines geriatrischen

Handlungsbedarfs im Aufnahmeprozess,

- Medikationsanamnese inklusive Plausibilitätsprüfung zum Aufnahmezeitpunkt,

- geriatrisches Fachwissen und soziale Kompetenzen des betreuenden Teams im Umgang mit geriatrischen Patienten der Notaufnahme,

- Identifikation möglicher Sturzrisikofaktoren.

Diese und weitere Punkte wurden auch in dem vor Kurzem fertiggestellten Curriculum für geriatrische Notfallmedizin der Special Interest Group Geriatric Emergency Medicine (GEM) der European Union Geriatric Medicine Society (EUGMS) in Kooperation mit der European Society for Emergency Medicine (EUSEM) aufgenommen. In diesem werden Kompetenzen, die in der Betreuung älterer (>65 Jahre) Notfallpatienten von Bedeutung sind, aufgeführt und erläutert [31].

\section{Weiterer Handlungsbedarf - Versorgungsforschung}

Der Erstkontakt aller Notfallpatienten muss auf der Grundlage von validierten Triage-Instrumenten erfolgen [32].

Die Identifizierung eines geriatrischen Handlungsbedarfs im weiteren Aufnahmeprozess wird von den beteiligten Fachgesellschaften als ein zentraler Punkt bewertet [33-35]. Bisher existiert leider kein Screening-Instrument, wel- 
ches den Belangen der klinischen Akutund Notfallmedizin und der Geriatrie bezüglich Validität und Praktikabilität Stand halten.

Um praktikable und aussagekräftige Risikofaktoren zuverlässig zu identifizieren, ist eine weiterführende Versorgungsforschung im Bereich der notfallmedizinischen Versorgung geriatrischer Patienten dringend zu fordern.

Die Versorgung geriatrischer Patienten muss zwar dem notfallmedizinischen Arbeitsablauf, aber auch den besonderen Charakteristika dieser Patientengruppe gerecht werden. Hierfür bedarf es klarer Zielkriterien (Einfluss auf die Lebensqualität, Einfluss auf die kognitive und physische Funktionalität, Einfluss auf Komplikationen im weiteren Behandlungsverlauf) und der Vermeidung von Überund Unterversorgung.

\section{Zentrale Forderungen}

1. Der Erstkontakt in den Notaufnahmen ist für alle Patienten durch ein validiertes Triage-Instrument sicherzustellen.

2. Bereits in der Notaufnahme muss ein obligatorischer Ausschluss eines Delirs erfolgen.

3. Bereits entwickelte Qualitätsindikatoren für die Versorgung geriatrischer Notfallpatienten sind verbindlich festzulegen.

4. Vulnerabilität und Risikofaktoren der geriatrischen Patienten müssen systematisch mit noch zu entwickelnden Screening-Instrumenten erfasst werden.

5. Weiterführende Versorgungsforschung im Bereich der Akut- und Notfallmedizin geriatrischer Patienten ist zu fordern.

\section{Korrespondenzadresse}

\section{PD Dr. med. K. Singler}

Institut für Biomedizin des Alterns, FriedrichAlexander-Universität Erlangen-Nürnberg Kobergerstr. 60, 90408 Nürnberg, Deutschland katrin.singler@klinikum-nuernberg.de

\section{Einhaltung ethischer Richtlinien}

Interessenkonflikt. K. Singler, H. Dormann, C. Dodt, H.J. Heppner, R. Püllen, M. Burkhardt, W. Swoboda, R.E. Roller-Wirnsberger, G. Pinter, P. Mrakund T.Münzer geben an, dass kein Interessenkonflikt besteht.

Dieser Beitrag beinhaltet keine von den Autoren durchgeführten Studien an Menschen oder Tieren.

\section{Literatur}

1. Statistisches Bundesamt. Alter im Wandel: Ältere Menschen in Deutschland und der EU-2012. Statistisches Bundesamt, Wiesbaden. https://www.destatis.de/DE/Publikationen/ Thematisch/Bevoelkerung/Bevoelkerungsstand/ BlickpunktAeltereMenschen. Zugegriffen: 30. Dezember 2015

2. http://www.dggeriatrie.de/nachwuchs/91-wasist-geriatrie.html. Zugegriffen: 30. Dezember 2015

3. http://www.geriatrie-online.at Zugegriffen: 30. Dezember 2015

4. Sieber CC (2007) Der ältere Patient - wer ist das? Internist (Berl) 48:1190-1194

5. Singler K, Christ M, Sieber C, Gosch M, Heppner HJ (2011) Geriatric patients in emergency and intensive care medicine. Internist (Berl) 52(8):934-938

6. Aminzadeh F, Dalziel WB (2002) Older adults in the emergency department: a systematic review of patterns of use, adverse outcomes, and effectiveness of interventions. Ann Emerg Med 39(3):238-247

7. Groening M, Grossmann F, Hilmer T, Schuster S, Singler K, Somasundaram R, Wilke P (2015) ÄLPANO - erste Ergebnisse der DGINA-Umfrage zur Versorgung älterer Notfallpatienten. Notf + Rettungsmed 18(Suppl 1):13

8. Grossmann FF, Zumbrunn T, Frauchiger A et al (2012) At risk of undertriage? Testing the performance and accuracy of the emergency severity index in older emergency department patients. Ann Emerg Med 60:317-325.e3

9. Hortmann M, Singler K, Geier F, Christ M (2015) Recognition of infections in elderly emergency patients. ZGerontol Geriatr 48:601-607

10. Nemec M, Koller MT, Nickel CH et al (2010) Patients presenting to the emergency department with non-specific complaints: the Basel Non-specific Complaints (BANC) study. Acad Emerg Med 17:284-292

11. Carpenter CR, DesPain B, Keeling TK, Shah M, Rothenberger M (2011) The six-item screener and AD8 for the detection of cognitive impairment in geriatric emergency department patients. Ann Emerg Med 57:653-661

12. Han JH, Wilber ST (2013) Altered Mental Status in Older Emergency Department Patients. Clin Geriatr Med 29(1):101-136

13. Samara N, Chevalley T, Samaras D, Gold G (2010) Older patients in the emergency department: a review. Ann Emerg Med 56(3):261-269

14. BarnettK, Mercer S, Norbury M, Watt G, Wyke S, Guthrie B (2012) The epidemiology of multimorbidity in a large cross-sectional dataset: implications for health care, research and medical education. Lancet 380:37-43

15. Singler K, Thiem U, ChristMetal (2014) Aspects and assessment of delirium in old age. First data from a German interdisciplinary emergency department. ZGerontol Geriatr 47:680-685
16. Gower LEJ, Gatewood MO, Kang CS (2012) Emergency department management of delirium in the elderly. Western JEmerg Med 13(2):194-201

17. LaMantia MA, Messina FC, Hobgood CD, Miller DK (2014) Screening for Delirium in the Emergency Department: ASystematic Review. Ann Emerg Med 63(5):551-560

18. Dormann H, Sonst A, Müller F et al (2013) Adverse drug events in older patients admitted as an emergency: the role of potentially inappropriate medication in elderly people (PRISCUS). Dtsch Arztebl Int 110:213-219

19. Singal BM, Hedges JR, Rosseau EW et al (1992) Geriatric patient emergency visits. Part 1: comparison of visits by geriatric and younger patients. Ann Emerg Med 21(7):802-807

20. Biber R, Bail HJ, Sieber C, Weis $P$, Christ M, Singler K (2013) Correlation between age, emergency department length of stay and hospital admission rate in emergency department patients aged $\geq 70$ years. Gerontology 59:17-22

21. Kada O, Brunner E, Likar R, Pinter G, Leutgeb I, Francisci N, Pfeiffer B, Janig H (2011) Vom Pflegeheim ins Krankenhaus und wieder zurück - Eine multimethodale Analyse von Krankenhaustransporten aus Alten- und Pflegeheimen. Z Evid Fortbild Qual GesundheitswWesen (ZEFQ) 105:714-722

22. Terrell KM, Hustey FM, Hwang U, Gerson LW, Wenger NS, Miller DK (2009) Society for Academic Emergency Medicine (SAEM) Geriatric Task Force. Quality indicators for geriatric emergency care. Acad Emerg Med 16:441-449

23. CarpenterCR, Heard K, Wilber S, Ginde AA, StifflerK, Gerson LW, Wenger NS, Miller DK (2011) Society for Academic Emergency Medicine (SAEM) Geriatric Task Force Research priorities for high-quality geriatric emergency care: medication management, screening, and prevention and functional assessment. Acad Emerg Med 18:644-654

24. Carpenter CR, Shah MN, Hustey FM, Heard K, Gerson LW, Miller DK (2011) High yield research opportunities in geriatric emergency medicine: prehospital care, delirium, adverse drug events, and falls. J Gerontol A Biol Sci Med Sci 66:775-783

25. Schnitker LM, Martin-Khan M, BurkettEet al (2015) Process quality indicators targeting cognitive impairment to support quality of care for older people with cognitive impairment in emergency departments. Acad Emerg Med 22:285-298

26. Schnitker LM, Martin-Khan M, BurkettE et al (2015) Structural quality indicators to support quality of care for older people with cognitive impairment in emergency departments. Acad Emerg Med 22:273-284

27. Bramesfeld A, Willms G (2014) Gesetzliche Qualitätssicherung in der medizinischen Versorgung: Stand und Weiterentwicklung - Sektorenübergreifende Qualitätssicherung nach § 137a SGBV.Public Health Forum. doi:10.1016/j.phf.2014.03.014

28. SQG - Sektorenübergreifende Qualität im Gesundheitswesen. http://www.sqg.de/front_content. php?idcat=84. Zugegriffen:30. Dezember 2015

29. BQS, Institut für Qualität und Patientensicherheit, Innovationen QUALIFY-Instrument; http://www.bqs.de/leistungen/beratung-undwissenschaftliche-studien. Zugegriffen: 24 . August 2016

30. Schuster S, Willam C, Dormann H (2015) GeriQ - Entwicklung von Qualitätsindikatoren für eine geriatrische Notfallversorgung (QÖP-V01). Notfall Rettungsmed 18(Suppl 1):7 (Abstracts zu Vorträgen und Postern der 10. Jahrestagung der Deutschen Gesellschaft interdisziplinäre Notfallund Akutmedizin) 
31. Abdelouahab B, Conroy S, Graham CA (2016) The European curriculum for geriatric emergency medicine. Eur JEmerg Med 2016;23:239

32. Christ M, Grossmann F, Winter D, Bingisser R, Platz $E$ (2010) Modern triage in the emergency department. Dtsch Arztebl Int 107:892-898

33. Carpenter CR, Shelton E, Fowler S et al (2015) Risk factors and screening instruments to predict adverse outcomes for undifferentiated older emergency department patients: a systematic review and meta-analysis. Acad Emerg Med 22:1-21

34. Hwang U, Carpenter C (2016) Assessing geriatric vulnerability for post emergency department adverse outcomes: challenges abound while progress is slow. Emerg Med J 33:2-3

35. Thiem U, Greuel HW, Reingräber A, Koch-Gwinner $P$, Püllen R, Heppner HJ, Pfisterer M (2012) Consensus for the identification of geriatric patients in the emergency care setting in Germany. Z Gerontol Geriatr 45:310-314

\section{Schnellere Herzinfarkt-Dia- gnose hilft Leben zu retten}

Die aktuellen Leitlinien der Deutschen Gesellschaft für Kardiologie empfehlen bei Verdacht auf Herzinfarkt, sofort bei der Aufnahme des Patienten und dann nochmals nach drei Stunden per Bluttest Troponin I zu messen. Nach derzeitigen Standards gelten Troponin-I-Werte über $27 \mathrm{ng} / \mathrm{l}$ als erhöht. Im UHZ wurde in den vergangenen Jahren eine Alternative entwickelt und erprobt. „Der neue, hochsensitive Troponin-I-Test liefert viel rascher Ergebnisse und entdeckt auch viel niedrigere Troponin-I-Werte, die aber eine wichtige Rolle für das kardiovaskuläre Risiko spielen dürften", erklärt PD Dr. Dirk Westermann, Leiter der sog. BACCStudie („Biomarkers in Acute Cardiac Care“), deren Ergebnisse jetzt im Fachjournal JAMA Cardiology veröffentlicht wurden.

In die Studie wurden 1.040 Patienten mit einem Durchschnittsalter von 65 Jahren eingeschlossen, die mit akutem Brustschmerz und Herzinfarkt-Verdacht in der Notaufnahme des UKE betreut wurden. Es wurden sowohl der herkömmliche Troponin-I-Test mit dreistündiger Wartezeit als auch das neue Testverfahren durchgeführt. Auf Basis des Standardverfahrens wurde bei 184 Patienten ein Herzinfarkt diagnostiziert und eine weitere stationäre Behandlung veranlasst, die anderen wurden nach Hause entlassen. Alle Patienten wurden über 12 Monate weiter beobachtet.

Beim Vergleich der Ergebnisse beider Testverfahren stellte sich heraus, dass bereits ein Grenzwert von nur 6 ng/l Troponin I, bei der Aufnahme und nach einer Stunde gemessen, zuverlässiger den Ausschluss eines Herzinfarkts ermöglicht als die bisher empfohlenen $27 \mathrm{ng} / \mathrm{l}$ mit Wiederholungsmessung nach drei Stunden.

Der neue Algorithmus mit dem niedrigeren Grenzwert wurde über die UKE-eigene Studie hinaus anhand der Daten zweier weiterer großer Studien, an der mehr als 4.000 Patienten mit akutem Brustschmerz und Herzinfarkt-Verdacht beteiligt waren, überprüft und bestätigt. Diese Studie, so Prof. Blankenberg, Direktor der Klinik für Allgemeine und Interventionelle Kardiologie im Universitären Herzzentrum (UHZ), wird Einfluss auf eine zukünftige Änderung der Leitlinien zur Diagnose des akuten Myokardinfarktes besitzen.

Neumann et al. Diagnosis of Myocardial Infarction Using a High-Sensitivity Troponin I 1-Hour Algorithm. JAMA Cardiology. Published online June 1,2016; doi:10.1001/jamacardio.2016.0695

Quelle: Universitätsklinikum Hamburg-Eppendorf (UKE) Martinistraße 52 